This item was submitted to Loughborough's Research Repository by the author.

Items in Figshare are protected by copyright, with all rights reserved, unless otherwise indicated.

\title{
Deformations of the root systems and new solutions to generalised WDVV
} \section{equations}

PLEASE CITE THE PUBLISHED VERSION

LICENCE

CC BY-NC-ND 4.0

\section{REPOSITORY RECORD}

Veselov, A.P.. 2019. "Deformations of the Root Systems and New Solutions to Generalised WDVV Equations". figshare. https://hdl.handle.net/2134/830. 


\title{
Deformations of the root systems and new solutions to generalised WDVV equations
}

\author{
March 17, 1999
}

\author{
A.P.Veselov \\ Department of Mathematical Sciences, Loughborough University, \\ Loughborough, Leicestershire, LE 11 3TU, UK \\ Landau Institute for Theoretical Physics, Kosygina 2, \\ Moscow, 117940, Russia \\ e-mail: A.P.Veselov@lboro.ac.uk,
}

\begin{abstract}
A special class of solutions to the generalised WDVV equations related to a finite set of covectors is investigated. Some geometric conditions on such a set which guarantee that the corresponding function satisfies WDVV equations are found ( $\vee$-conditions). These conditions are satisfied for all root systems and their special deformations discovered in the theory of the Calogero-Moser systems by O.Chalykh, M.Feigin and the author. This leads to the new solutions for the generalized WDVV equations.
\end{abstract}

\section{Introduction.}

The generalised WDVV (Witten-Dijgraaf-Verlinde-Verlinde) equations are the following overdetermined system of nonlinear differential equations:

$$
F_{i} F_{k}^{-1} F_{j}=F_{j} F_{k}^{-1} F_{i}, \quad i, j, k=1, \ldots, n,
$$

where $F_{m}$ is the $n \times n$ matrix constructed from the third partial derivatives of the unknown function $F=F\left(x^{1}, \ldots, x^{n}\right)$ :

$$
\left(F_{m}\right)_{p q}=\frac{\partial^{3} F}{\partial x^{m} \partial x^{p} \partial x^{q}},
$$


In this form these equations have been presented by A.Marshakov,A.Mironov and A.Morozov, who showed that the Seiberg-Witten prepotential in $N=2$ four-dimensional supersymmetric gauge theories satisfies this system [1]. Originally these equations appeared in a slightly different form as associativity conditions in topological field theory (see [2]).

In the paper [3] for any classical root system and in [4] for any root system $\mathcal{R} \in \mathbf{R}^{n}$ of a semisimple Lie algebra of rank $n$ it has been shown that the function

$$
F(x)=\frac{1}{4} \sum_{\alpha \in \mathcal{R}}(\alpha, x)^{2} \log (\alpha, x)^{2}
$$

satisfies the WDVV equations $(1,2)$.

Our first observation is that this is actually true for all the Coxeter configurations related to any finite reflection group.

The second, main observation is that the function (3) satisfies WDVV equation also for certain deformations of the root systems discovered by O.Chalykh, M.Feigin and the author $[5,6,7]$. The corresponding families of the solutions to WDVV equations have the form

$$
F=\sum_{i<j}^{n}\left(x_{i}-x_{j}\right)^{2} \log \left(x_{i}-x_{j}\right)^{2}+\frac{1}{m} \sum_{i=1}^{n} x_{i}^{2} \log x_{i}^{2}
$$

with an arbitrary real value of the parameter $m$ and

$$
\begin{gathered}
F=k \sum_{i<j}^{n}\left[\left(x_{i}+x_{j}\right)^{2} \log \left(x_{i}+x_{j}\right)^{2}+\left(x_{i}-x_{j}\right)^{2} \log \left(x_{i}-x_{j}\right)^{2}\right]+ \\
+\sum_{i=1}^{n}\left[\left(x_{i}+x_{n+1}\right)^{2} \log \left(x_{i}+x_{n+1}\right)^{2}+\left(x_{i}-x_{n+1}\right)^{2} \log \left(x_{i}-x_{n+1}\right)^{2}\right]+ \\
+4 m \sum_{i=1}^{n} x_{i}^{2} \log x_{i}^{2}+4 l x_{n+1}^{2} \log x_{n+1}^{2},
\end{gathered}
$$

where the real parameters $k, m, l$ satisfy the only relation

$$
k(2 l+1)=2 m+1 .
$$

When $m=1$ the formula (4) gives the well-known solution to WDVV equations, corresponding to the leading perturbative approximation to the exact Seiberg-Witten prepotential for the gauge group $S U(n+1)$ (see [1]). For the general $m$ it corresponds to the deformation $A_{n}(m)$ of the root system $A_{n}$ related to the Lie algebra $s u(n+1)$ (see [5] and below). Corresponding solution (4) has been found earlier in [3] (see the formula (3.15) and the calculations after that) ${ }^{1}$ but the fact that it is related to the configurations with non-Coxeter geometry has not been observed that time.

The second family of the solutions to WDVV equations (5) seems to be new. When $k=m=l=1$ they correspond to the root system $C_{n+1}$, in the general case - to its deformation $C_{n+1}(m, l)$ (see [7] and below).

\footnotetext{
${ }^{1}$ I am grateful to Andrei Marshakov who pointed out this to me.
} 
Actually I will describe some geometric conditions ( $\vee$-conditions) on the set of the covectors $\alpha$, which guarantee that the corresponding function (3) satisfies the generalised WDVV equations. The roots systems and their deformations mentioned above satisfy these conditions. The classification of all $\vee$-systems is an interesting geometrical problem, which seems to be new.

\section{$1 \quad \vee$-systems and particular solutions to WDVV equations.}

Let's first remind the following observation from $[1,8]$ that WDVV equations (1), (2) are equivalent to the equations

$$
F_{i} G^{-1} F_{j}=F_{j} G^{-1} F_{i}, \quad i, j=1, \ldots, n,
$$

where $G=\sum_{k=1}^{n} \eta^{k} F_{k}$ is any particular invertible linear combination of $F_{i}$ with the coefficients, which may depend on $x$. Introducing the matrices $\check{F}_{i}=G^{-1} F_{i}$ one can rewrite $(7)$ as the commutativity relations

$$
\left[\check{F}_{i}, \check{F}_{j}\right]=0, \quad i, j=1, \ldots, n,
$$

Let us consider now the following particular class of the solutions to these equations.

Let $V$ be a real linear vector space of dimension $n, V^{*}$ be its dual space consisting of the linear functions on $V$ (covectors), $\mathfrak{A}$ be a finite set of noncollinear covectors $\alpha \in V^{*}$. Most of the constructions below work also in the complex vector spaces, so we will discuss this case as well.

Consider the following function on $V$ :

$$
F^{\mathfrak{A}}=\sum_{\alpha \in \mathfrak{A}}(\alpha, x)^{2} \log (\alpha, x)^{2}
$$

where $(\alpha, x)=\alpha(x)$ is the value of covector $\alpha \in V^{*}$ on a vector $x \in V$. For any basis $e_{1}, \ldots, e_{n}$ we have the corresponding coordinates $x^{1}, \ldots, x^{n}$ in $V$ and the matrices $F_{i}$ defined according to $(2)$. In a more invariant form for any vector $a \in V$ one can define the matrix

$$
F_{a}=\sum_{i=1}^{n} a^{i} F_{i} .
$$

By a straightforward calculation one can check the following

Lemma. For a function (9) $F_{a}$ is the matrix of the following bilinear form on $V$

$$
F_{a}^{\mathfrak{A}}=\sum_{\alpha \in \mathfrak{A}} \frac{(\alpha, a)}{(\alpha, x)} \alpha \otimes \alpha,
$$

where $\alpha \otimes \beta(u, v)=\alpha(u) \beta(v)$ for any $u, v \in V$ and $\alpha, \beta \in V^{*}$. 
Now let us choose $G^{\mathfrak{A}}$ as $F_{x}^{\mathfrak{A}}$ :

$$
G^{\mathfrak{A}}=\sum_{i=1}^{n} x^{i} F_{i} .
$$

Then, according to the lemma $G^{\mathfrak{A}}$ is a matrix of the bilinear form

$$
G^{\mathfrak{A}}=\sum_{\alpha \in \mathfrak{A}} \alpha \otimes \alpha, G^{\mathfrak{A}}(u, v)=\sum_{\alpha \in \mathfrak{A}}(\alpha, u)(\alpha, v) .
$$

Notice that $G^{\mathfrak{A}}$ does not depend on $x$.

We will assume that $G^{\mathfrak{A}}$ is non-degenerate, which in the real case means that the covectors $\alpha \in \mathfrak{A}$ generate $V^{*}$. This means that the natural linear mapping $\varphi_{\mathfrak{A}}: V \rightarrow V^{*}$ defined by the formula

$$
\left(\varphi_{\mathfrak{A}}(u), v\right)=G^{\mathfrak{A}}(u, v), u, v \in V
$$

is invertible. We will denote $\varphi_{\mathfrak{A}}^{-1}(\alpha), \alpha \in V^{*}$ as $\alpha^{\vee}$. By definition, for any $v \in V$

$$
(\alpha, v)=\sum_{\beta \in \mathfrak{A}}\left(\alpha, \beta^{\vee}\right)(\beta, v) .
$$

Now let us define the operator

$$
\check{F}_{a}^{\mathfrak{A}}=\sum_{\alpha \in \mathfrak{A}} \frac{(\alpha, a)}{(\alpha, x)} \alpha^{\vee} \otimes \alpha
$$

According to (8) the WDVV equations $(1,2)$ for the function (9) are equivalent to

$$
\left[\check{F}_{a}^{\mathfrak{A}}, \check{F}_{b}^{\mathfrak{A}}\right]=0
$$

for any $a, b \in V$. A simple calculation shows that (15) can be rewritten as

$$
\sum_{\alpha \neq \beta, \alpha, \beta \in \mathfrak{A}} \frac{G^{\mathfrak{A}}\left(\alpha^{\vee}, \beta^{\vee}\right) B_{\alpha, \beta}(a, b)}{(\alpha, x)(\beta, x)} \alpha \wedge \beta \equiv 0,
$$

where

$$
\alpha \wedge \beta=\alpha \otimes \beta-\beta \otimes \alpha
$$

and

$$
B_{\alpha, \beta}(a, b)=\alpha \wedge \beta(a, b)=\alpha(a) \beta(b)-\alpha(b) \beta(a) .
$$

Thus the WDVV equations for the function (9) are equivalent to the conditions (16) to be satisfied for any $x, a, b \in V$.

Notice that WDVV equations $(1,2)$ and, therefore, the conditions (16) are obviously satisfied for any two-dimensional configuration $\mathfrak{A}$. This fact and the structure of the relation (16) motivate the following notion of the $\vee$-systems. 
Remind first that for a pair of bilinear forms $F$ and $G$ on the vector space $V$ one can define an eigenvector $e$ as the kernel of the bilinear form $F-\lambda G$ for a proper $\lambda$ :

$$
(F-\lambda G)(v, x)=0
$$

for any $v \in V$. When $G$ is non-degenerate $e$ is the eigenvector of the corresponding operator $\check{F}=G^{-1} F$ :

$$
\check{F}(e)=G^{-1} F(e)=\lambda e .
$$

Now let $\mathfrak{A}$ be as above any finite set of non-collinear covectors $\alpha \in V^{*}, G=G^{\mathfrak{A}}$ be the corresponding bilinear form (12), which is assumed to be non-degenerate, $\alpha^{\vee}$ are defined by (13). Define now for any two-dimensional plane $\Pi \subset V^{*}$ a form

$$
G_{\Pi}^{\mathfrak{A}}(x, y)=\sum_{\alpha \in \Pi \cup \mathfrak{A}}(\alpha, x)(\alpha, y) .
$$

Definition. We will say that $\mathfrak{A}$ satisfies the $\vee$-conditions if for any plane $\Pi \in V^{*}$ the vectors $\alpha^{\vee}, \alpha \in \Pi \cup \mathfrak{A}$ are the eigenvectors of the pair of the forms $G^{\mathfrak{A}}$ and $G_{\Pi}^{\mathfrak{A}}$. In this case we will call $\mathfrak{A}$ as $\vee$-system.

The $\vee$-conditions can be written explicitly as

$$
\sum_{\beta \in \Pi \cap \mathfrak{A}} \beta\left(\alpha^{\vee}\right) \beta^{\vee}=\lambda \alpha^{\vee}
$$

for any $\alpha \in \Pi \cap \mathfrak{A}$ and some $\lambda$, which may depend on $\Pi$ and $\alpha$.

If the plane $\Pi$ contains no more that one vector from $\mathfrak{A}$ then this condition is obviously satisfied, so the $\vee$-conditions should be checked only for a finite number of planes $\Pi$.

If the plane $\Pi$ contains only two covectors $\alpha$ and $\beta$ from $\mathfrak{A}$ then the condition (18) means that $\alpha^{\vee}$ and $\beta^{\vee}$ are orthogonal with respect to the form $G^{\mathfrak{A}}$ :

$$
\beta\left(\alpha^{\vee}\right)=G^{\mathfrak{A}}\left(\alpha^{\vee}, \beta^{\vee}\right)=0 .
$$

If the plane $\Pi$ contains more that two covectors from $\mathfrak{A}$ this condition means that $G$ and $G_{\Pi}$ restricted to the plane $\Pi^{\vee} \subset V$ are proportional:

$$
\left.G_{\Pi}\right|_{\Pi \vee}=\left.\lambda(\Pi) G\right|_{\Pi \vee}
$$

Theorem 1. Let $\mathfrak{A}$ be any $\vee$-system, then the function (9) satisfies the $W D V V$ equations (1).

Proof. It is enough to prove that

$$
\sum_{\alpha, \beta \in \Pi \cap \mathfrak{A}} \frac{G^{\mathfrak{A}}\left(\alpha^{\vee}, \beta^{\vee}\right) B_{\alpha, \beta}(a, b)}{(\alpha, x)(\beta, x)} \alpha \wedge \beta \equiv 0
$$

for any plane $\Pi \in V^{*}$. When $\Pi \cap \mathfrak{A}$ consists only of two covectors $\alpha$ and $\beta$ this follows from $G^{\mathfrak{A}}\left(\alpha^{\vee}, \beta^{\vee}\right)=0$, which is the $\vee$-condition in this case. If 
$\Pi \cap \mathfrak{A}$ consists of more that two covectors this relation is proportional to the corresponding relation for the function

$$
\left.F_{\Pi}^{\mathfrak{A}}\right|_{\Pi^{\vee}}=\left.\sum_{\alpha \in \mathfrak{A} \cap \Pi}(\alpha, x)^{2} \log (\alpha, x)^{2}\right|_{\Pi^{\vee}}
$$

Since $\Pi^{\vee}$ is two-dimensional this is obviously satisfied.

Remark. Elementary geometric arguments show that in the real case the inverse statement is also true, i.e. if the function (9) satisfies WDVV equations (1) then $\mathfrak{A}$ must be a $\vee$-system. Probably this is true in the complex space as well but the proof is still to be found.

\section{Examples of $\vee$-systems: root systems and their deformations.}

Let $V$ be now Euclidean vector space with a scalar product (, ), and $G$ be any irreducible finite group generated by orthogonal reflections with respect to some hyperplanes (Coxeter groups [9]). Let $\mathcal{R}$ be a set of normal vectors to the reflection hyperplanes of $G$. We will not fix the length of the normals but assume that $\mathcal{R}$ is invariant under the natural action of $G$ and contains exactly two normal vectors for any such hyperplane. Let us choose from each such pair of vectors one of them and form the system $\mathcal{R}_{+}$:

$$
\mathcal{R}=\mathcal{R}_{+} \cup\left(-\mathcal{R}_{+}\right)
$$

Usually $\mathcal{R}_{+}$is chosen simply by taking from $\mathcal{R}$ vectors which are positive with respect to some linear form on $V$. We will call a system $\mathcal{R}_{+}$as Coxeter system and the vectors from $\mathcal{R}_{+}$as roots.

Theorem 2. Any Coxeter system $\mathcal{R}_{+}$is a $\vee$-system.

Proof is very simple. First of all the form (12) in this case is proportional to the euclidean structure on $V$ because it is invariant under $G$ and $G$ is irreducible. By the same reason this is true for the form $G_{\Pi}(17)$ if the plane $\Pi$ contains more than two roots from $\mathcal{R}_{+}$. When $\Pi$ contains only two roots they must be orthogonal and therefore satisfy $\vee$-conditions.

Corollary. For any Coxeter system $\mathcal{R}_{+}$the function

$$
F=\sum_{\alpha \in \mathcal{R}_{+}}(\alpha, x)^{2} \log (\alpha, x)^{2}
$$

satisfy WDVV equations (1), (2).

Remark. The root systems of any semisimple Lie algebra are the particular examples of the Coxeter systems. In this case this result has been proven in [4]. Notice that even when $G$ is a Weyl group of some Lie algebra our formula (20) in general gives more solutions since we have not fixed the length of the roots. 
Remarkably enough that the theorem 2 is true also for the following deformations of the root systems found in the theory of the generalised Calogero-Moser systems in $[5,6,7]$.

Let us make first the following remark. One can consider the class of functions related to a formally more general situation when the covectors $\alpha$ have also some prescribed multiplicities $\mu_{\alpha}$

$$
F^{(\mathfrak{A}, \mu)}=\sum_{\alpha \in \mathfrak{A}} \mu_{\alpha}(\alpha, x)^{2} \log (\alpha, x)^{2} .
$$

But it is easy to see that this actually will give no new solutions because $F^{(\mathfrak{A}, \mu)}=$ $F^{\tilde{\mathfrak{A}}}+$ quadratic terms, where $\tilde{\mathfrak{A}}$ consists of covectors $\sqrt{\mu_{\alpha}} \alpha$.

The following configurations $A_{n}(m)$ and $C_{n+1}(m, l)$ have been introduced in $[5,6,7]$. They consist of the following vectors in $\mathbf{R}^{n+1}$ :

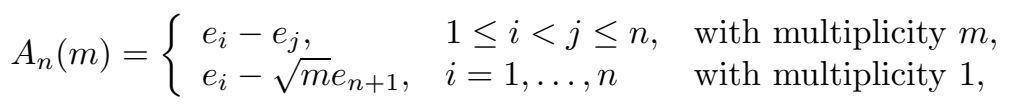

and

$$
C_{n+1}(m, l)=\left\{\begin{array}{lll}
e_{i} \pm e_{j}, & 1 \leq i<j \leq n, & \text { with multiplicity } k \\
2 e_{i}, & i=1, \ldots, n & \text { with multiplicity } m \\
e_{i} \pm \sqrt{k} e_{n+1}, & i=1, \ldots, n \\
2 \sqrt{k} e_{n+1} & \text { with multiplicity } l, & \text { with multiplicity } 1
\end{array}\right.
$$

where $k=\frac{2 m+1}{2 l+1}$.

When all the multiplicities are integer the corresponding generalisation of Calogero-Moser system is algebraically integrable, but usual integrability holds for any value of multiplicities (see $[5,6,7]$ ).

Notice that when $m=1$ the first configuration coincides with the classical root system of type $A_{n}$ and when $k=m=l=1$ the second configuration is the root system of type $C_{n+1}$. So these families can be considered as the special deformations of these roots systems.

Theorem 3. For the deformed root systems $A_{n}(m)$ and $C_{n+1}(m, l)$ with arbitrary $m$ and $l$ the function (21) satisfies WDVV equation.

This follows from the simple check that the sets

$$
\tilde{A}_{n}(m)= \begin{cases}\sqrt{m}\left(e_{i}-e_{j}\right), & 1 \leq i<j \leq n, \\ e_{i}-\sqrt{m} e_{n+1}, & i=1, \ldots, n\end{cases}
$$

and

$$
\tilde{C}_{n+1}(m, l)= \begin{cases}\sqrt{k} e_{i} \pm \sqrt{k} e_{j}, & 1 \leq i<j \leq n \\ 2 \sqrt{m} e_{i}, & i=1, \ldots, n \\ e_{i} \pm \sqrt{k} e_{n+1}, & i=1, \ldots, n \\ 2 \sqrt{k l} e_{n+1}, & \end{cases}
$$


with $k=\frac{2 m+1}{2 l+1}$ satisfy the $\vee$-conditions. Making a suitable linear transformation one can rewrite these families in the following, more convenient way:

$$
\tilde{A}_{n}(m)= \begin{cases}e_{i}-e_{j}, & 1 \leq i<j \leq n, \\ \frac{1}{\sqrt{m}} e_{i}, & i=1, \ldots, n\end{cases}
$$

and

$$
\tilde{C}_{n+1}(m, l)= \begin{cases}\sqrt{k}\left(e_{i} \pm e_{j}\right), & 1 \leq i<j \leq n, \\ 2 \sqrt{m} e_{i}, & i=1, \ldots, n \\ e_{i} \pm e_{n+1}, & i=1, \ldots, n \\ 2 \sqrt{l} e_{n+1}, & \end{cases}
$$

where again $k=\frac{2 m+1}{2 l+1}$.

Corresponding functions $F$ have the form (4), (5) written in the Introduction.

Corollary. The functions $F$ given by the formulas (4), (5) satisfy WDVV equations.

At the moment I have no satisfactory explanation why the deformed root systems arisen in the theory of the generalised Calogero-Moser problems turned out to be $\vee$-systems. It may be that it is a common geometrical property of all the so-called locus configurations [7]. In this connection I'd like to mention that $\vee$-systems can be naturally defined in a complex vector space. Their classification seems to be very interesting and important problem.

\section{$3 \quad$ Final remarks: Frobenius structures.}

Boris Dubrovin has proposed an elegant geometric axiomatisation of the original WDVV equations introducing a notion of Frobenius manifold (see [2]). Although the functions (9) related to the $\vee$-systems do not satisfy all the properties [2] to determine the structure of Frobenius manifold (the unity vector field is not covariantly constant in this case) they determine the so-called almost Frobenius structures. Dubrovin communicated to me about the calculations he has made some time ago, which indicate that these structures in the Coxeter case are in some concrete sense (discovered by Dubrovin) are dual to the Frobenius structures on the spaces of orbits of Coxeter groups he described in [10]. The natural question is whether this can be generalised for the non-Coxeter $\vee$-systems and what are the corresponding dual Frobenius structures. We hope to come back to these questions soon. 


\section{Acknowledgements.}

I am grateful to M.Feigin for the critical remarks and suggestions and to B.Dubrovin for very interesting discussions during the conference on Random Matrices and Integrable Systems (MSRI,Berkeley, February 22-26, 1999) where this material has been first presented.

\section{References}

[1] A.Marshakov, A.Mironov, and A.Morozov $W D V V$-like equations in $N=2$ SUSY Yang-Mills theory. Phys.Lett. B, 389 (1996), 43-52, hep-th/9607109.

[2] B.Dubrovin Geometry of 2D topological field theories. Nucl.Phys., B352 (1992), 627, hep-th/9407018.

[3] A.Marshakov, A.Mironov, and A.Morozov More evidence for the WDVV equations in N=2 SUSY Yang-Mills theories. hep-th/9701123.

[4] R.Martini, P.K.H.Gragert Solutions of WDVV equations in Seiberg-Witten theory from root systems. J.of Nonlinear Math.Physics, 6 (1) (1999), 1-4.

[5] A.P. Veselov, M.V. Feigin, O.A. Chalykh New integrable deformations of quantum Calogero - Moser problem. Usp. Mat. Nauk 51 (3) (1996), 185186.

[6] O.A. Chalykh, M.V. Feigin, A.P. Veselov New integrable generalizations of Calogero-Moser quantum problem. J. Math. Phys 39 (2) (1998), 695-703.

[7] O.A. Chalykh, M.V. Feigin, A.P. Veselov. Multidimensional Baker-Akhiezer Functions and Huygens' Principle. Submitted to Commun. Math. Physics, math-ph/9903019.

[8] A.Marshakov, A.Mironov, A.Morozov WDVV equations from algebra of forms. Mod.Phys.Lett A12 (1997) 773, hep-th/9701014.

[9] N. Bourbaki, Groupes et algèbres de Lie. Chap. VI, Masson, 1981

[10] B.Dubrovin Differential Geometry of the space of orbits of a Coxeter group. hep-th/9303152. 